Veritas日 Scientia

Vol. $9 . \mathrm{N}^{\circ} 2$

Julio - Diciembre del 2020

ISSN Edición Online: 2617-0639

https://doi.org/10.47796/ves.v9i2.392

Artículo Original

\title{
RELACIÓN ENTRE AUTOEFICACIA ACADÉMICA Y AUTORREGULACIÓN DEL APRENDIZAJE EN ESTUDIANTES DE LA CARRERA DE TECNOLOGÍA MÉDICA
}

\author{
RELATIONSHIP BETWEEN ACADEMIC SELF-EFFICACY AND SELF-REGULATION \\ OF LEARNING IN STUDENTS OF THE MEDICAL TECHNOLOGY CAREER
}

Sandro Antonio Luna Vargas ${ }^{1}$

Rina María Álvarez Becerra ${ }^{2}$

Aceptado: $26 / 12 / 2019$

Publicado online: $14 / 12 / 2020$

\begin{abstract}
RESUMEN
El presente estudio tuvo como objetivo determinar si existe relación entre autoeficacia académica y autorregulación del aprendizaje en estudiantes de la carrera de tecnología médica de la Universidad Privada de Tacna. La población estuvo conformada por todos los estudiantes de la escuela de tecnología médica de los cuales solo decidieron participar 92. Se utilizó el Inventario de Expectativas de Autoeficacia Académica y el Inventario del Aprendizaje Autorregulado. Resultados: la autoeficacia académica un $57 \%$ se siente seguro, mientras que en el aprendizaje autorregulado el mayor porcentaje se concentra en un nivel medio con 50\%.Conclusión: al realizar la correlación de Spearman se encontró que existe una correlación positiva y moderada entre autoeficacia académica y el aprendizaje autorregulado en los estudiantes de la Escuela de Tecnología Médica de la Universidad Privada de Tacna en el año 2018 (Rho de Spearman ,479 ( $p$ valor 0.01)); al correlacionar la autoeficacia académica con las dimensiones de autorregulación del aprendizaje se llegaron a las siguientes conclusiones; autoeficacia académica con autorregulación de la estrategia ejecutiva mostro una correlación positiva y moderada (sig. Bilateral 0.01 y coeficiente de correlación ,449), al relacionarlo con autorregulación de la cognición mostro una correlación positiva y baja (sig. Bilateral 0.01 y coeficiente de correlación ,325), al relacionarlo con autorregulación de la motivación y afecto mostro una correlación positiva y baja (sig. Bilateral 0.01 y coeficiente de correlación ,398) y al relacionarlo con autorregulación del control del ambiente mostro una correlación positiva y baja (sig. Bilateral 0.01 y coeficiente de correlación ,385).
\end{abstract}

Palabras claves: autoeficacia académica, autorregulación del aprendizaje, aprendizaje autorregulado.

1 Tecnólogo Médico en Terapia Física y Rehabilitación, Magíster en Docencia Universitaria y Gestión Educativa. sandrolv.tmf@gmail.com. (10000-0001-7798-7999

2 Doctora en Educación con mención en gestión educativa. Académica de la Escuela de Posgrado de la Universidad Privada de Tacna, Universidad Nacional Jorge Basadre Grohmann, Universidad Católica Santa María de Arequipa, Especialidad Estadística para la investigación.. rina.alvarez.becerra@gmail.com. (1) 0000-0002-5455-6632. 


\begin{abstract}
The objective of this study was to determine whether there is a relationship between academic self-efficacy and self-regulation of learning in students of the medical technology career of the Private University of Tacna. The population consisted of all the students of the medical technology school of which only 92 decided to participate. The Inventory of Academic SelfEfficacy Expectations and the Self-Regulated Learning Inventory were used. Results: $57 \%$ academic self-efficacy feels safe, while in self-regulated learning the highest percentage is concentrated at an average level with $50 \%$. Conclusion: when performing Spearman's correlation it was found that there is a positive and moderate correlation between self-efficacy academic and self-regulated learning in the students of the School of Medical Technology of the Private University of Tacna in 2018 (Rho de Spearman, 479 ( $p$ value 0.01)); By correlating academic self-efficacy with the dimensions of self-regulation of learning, the following conclusions were reached; academic self-efficacy with self-regulation of the executive strategy showed a positive and moderate correlation (sig. Bilateral 0.01 and correlation coefficient, 449), when related to self-regulation of cognition showed a positive and low correlation (sig. Bilateral 0.01 and correlation coefficient, 325), when relating it to self-regulation of motivation and affection, it showed a positive and low correlation (sig. Bilateral 0.01 and correlation coefficient, 398) and when relating it to self-regulation of the control of the environment, it showed a positive and low correlation (sig. Bilateral 0.01 and correlation coefficient, 385).
\end{abstract}

Keywords: academic self-efficacy, self-regulation of learning, self-regulated learning.

\title{
INTRODUCCIÓN
}

La autoeficacia académica y la autorregulación del aprendizaje son dos aspectos importantes en los estudiantes, ya que permiten que el proceso de aprendizaje de desarrolle de manera adecuada e influye en otros aspectos como el rendimiento académico; por eso en las últimas décadas se han estado estudiando más a fondo; donde diferentes estudios han hallado relación entre estas dos variables en menor o mayor grado. Pero a nivel local son escasos los estudios que analizan estas variables, por lo cual este estudio tiene como finalidad analizar si existe relación entre la autoeficacia académica y la autorregulación del aprendizaje, así como también medir el nivel de estas dos variables en los alumnos de Tecnología Médica de la Universidad Privada de Tacna. Se observa que algunos alumnos en diferentes cursos y ciclos lograban desarrollar los ejercicios y cuestionarios durante la clase pero al cambiarles el contexto, como por ejemplo una práctica libre o una prueba; tenían dificultad acerca de las estrategias de aprendizaje que deberían utilizar, así como también influía mucho en algunos cuando el trabajo era grupal o individual, lo cual intervenía en los métodos para formular teorías y conceptos, por lo que a nivel emocional afectaba su capacidad para poder sentirse capaz de realizar los ejercicios o cumplir con los objetivos trazados en clase. Estando relacionado con los conceptos descritos anteriormente los cuales será las variables de este estudio.

Por lo cual en el siguiente trabajo se midió la autoeficacia académica y autorregulación del aprendizaje en los alumnos de la carrera de tecnología médica, así mismo hallar si existe relación relevante entre estas dos variables; y de esta manera poder saber cómo intervenir en la mejora y optimización de estas variables, permitiendo a la vez fijar un antecedente en nuestro contexto para futuros estudios. Por ende, la interrogante fue ¿Existe relación entre autoeficacia académica y autorregulación del 
aprendizaje en estudiantes de la carrera de tecnología médica de la Universidad Privada de Tacna en el 2018? La investigación es pertinente ya que al determinar el nivel de autoeficacia académica y la autorregulación del aprendizaje se identificará qué tan motivado se encuentra el estudiante durante el proceso de aprendizaje, si es frecuente el uso de estrategias en este proceso, si influye el contexto, (refiriéndonos al ambiente, el trabajo en equipo o individual y el uso de la información que se les proporciona), lo cual proporciona un panorama sobre las condiciones de nuestros estudiantes. Al obtener estos datos identificará los puntos fuertes y débiles, que al aplicar diferentes estrategias sopesaremos las deficiencias de haberlas, o en caso contrario de encontrar un buen nivel en ambas variables potenciarlas con la finalidad de obtener mejores resultados aún. Al mismo tiempo permitir el cumplimiento del "Artículo 87 deberes del docente, referente a orientar los estudiantes en su desarrollo profesional y/o académico“ (Ley Universitaria 30220) siendo un apoyo al servicio de tutoría ofrecido por la universidad privada de Tacna a fin de coordinar esfuerzos y acciones entre profesores y alumnos orientados a conseguir una óptima formación profesional. Para finalizar este estudio también pretende marcar un precedente para futuros estudios referidos a la autoeficacia académica y autorregulación del aprendizaje.

Alegre (2014) pudo observar que la autoeficacia académica, la autorregulación del aprendizaje y el rendimiento académico presentaron un coeficiente de correlación positivo y significativo, además entre la autoeficacia académica y la autorregulación del aprendizaje mostro una correlación positiva, significativa y moderada. Adanque (2016) tuvo como resultados que el 55\% de entrevistados se sienten seguros de ser autoeficaces académicamente, el 37,3\% muy seguro y el $7 \%$ poco seguro. En cuanto a las dimensiones; seguros de ser autoeficaces en cuanto a la producción mostro un 42,7\%, en cuanto a la autoeficacia de insumos 63,6\% muy seguros y seguros de ser autoeficaces en las actividades académicas de interacción el 60,9\%. Respecto al rendimiento académico la media es de 11,96 siendo la mínima nota 05 y la máxima 17. Con respecto a la relación entre las variables, estas muestran una relación positiva y débil, siendo las la dimensión de autoeficacia en las actividades académicas de interacción la única que se relacionaba con el rendimiento académico. Durán (2017) encontró el nivel de aprendizaje autorregulado predominantemente alto con un $63.68 \%$ de los estudiantes; en cuanto a la motivación emocional se encontró un nivel medio con 46,59\%. La relación entre la cognición y la inteligencia emocional es baja. La relación entre ejecución y la inteligencia emocional es moderada. La relación entre motivación y la inteligencia emocional es baja. La relación entre cognición y la inteligencia emocional es baja y positiva. La relación entre control de ambiente y la inteligencia emocional es baja y positiva.

Alarcón (2016) identificó que, a la aplicación de la escala de autoeficacia académica general, en un nivel alto se encuentra I $92 \%$ de los alumnos, en el nivel medio el 7,78\% y en el nivel bajo no se encontraron casos a identificar; mostrando los hombres una mínima diferencia sobre las mujeres en los niveles alto y medio de autoeficacia percibida. Respecto a la aplicación de la escala autorregulación y aprendizaje logró identificar que el 59\% tienen una valoración de 90.36 indicando un alto nivel de autorregulación en su aprendizaje, siendo ligeramente mayor que el de las mujeres. Por último, se estableció que existe una relación positiva con una magnitud débil entre la autoeficacia y autorregulación, si bien no hay un comportamiento geométrico, responde a una válida al alfa=0,05. Fernández y Bernardo (2011) muestran que mientras mayor es el nivel de autoeficacia en el uso de estrategias de autorregulación del aprendizaje, mayor es el uso de dichas estrategias y mayor el rendimiento académico de los alumnos. 
Aquino (2015) indica la existencia de una relación estadísticamente significativa entre la autoeficacia, la autorregulación y el rendimiento académico de los estudiantes de primer ingreso de la licenciatura de nutrición, así mismo se determinó que el promedio del nivel de autoeficacia de los estudiantes es ligeramente mayor que el de autorregulación y que ambas variables tienen una relación estadísticamente significativa. Hernández (2015) observó una relación significativa entre autoeficacia, auto regulación y rendimiento escolar, no se encontró relación estadísticamente significativa entre edad, genero, autorregulación y autoeficacia, en cuanto a rendimiento escolar se mostró una relación inversa entre la edad y el rendimiento escolar y el nivel de autoeficacia percibida está por debajo del nivel de autorregulación. Pool (2013) en su estudio los resultados se obtuvieron seis factores: autoeficacia percibida, problemas de concentración, metas de aprendizaje, estrategias metacognitivas, de dominio y de comprensión; identificando asociaciones entre autoeficacia percibida con metas de aprendizaje y problemas de concentración, también una relación negativa entre estas dos últimas variables. Los tres factores anteriores resultaron predictores de estrategias metacognitivas, de dominio y de comprensión. Para Barahona (2014) la correlación fue significativa entre autoeficacia y autorregulación, así como también entre la variable edad, ya que se observó que a mayor edad la autorregulación y con ello la autoeficacia. Para Hendriati (2013) la autoeficacia, la autorregulación del aprendizaje y los logros académicos están correlacionados positivamente, lo que implica que, si una de las tres variables experimenta un cambio positivo o negativo, las otras dos también experimentarán un cambio.

El objetivo finalmente fue establecer si existe relación entre autoeficacia académica y la autorregulación del aprendizaje en estudiantes de la carrera de tecnología médica de la Universidad Privada de Tacna en el 2018.

\section{Autoeficacia académica}

La autoeficacia tiene su origen en el inicio de la búsqueda de la Teoría del Aprendizaje Social en 1996, los estudios en ésta línea comienzan con Rottery, siendo continuados por Bandura en 1977 (Valverde Riascos, 2011) el cual lo perfiló dentro de su Teoría del Aprendizaje Social para poder identificar el impacto que tienen esas creencias sobre los resultados en el rendimiento académico, el aprendizaje, la elección vocacional y la motivación para aprender (Barahona Arévalo, 2014). Para Bandura (1997) la autoeficacia académica es considerada como la capacidad del estudiante o individuo para ejercer control sobre los propios procesos de pensamiento, la motivación y la acción, considerándolo como una característica que debe ser cultivada en el ser humano. Bandura (1997) presenta una interesante teoría social cognitiva, que para el tiempo que fue propuesta resultó de suma importancia para entender a mayor detalle los procesos de aprendizaje del ser humano, basándose no solamente en el área cognitiva, sino también en que las personas son agentes que inician las actividades movidos por la propia motivación que pueden generar en su interior (intrínseco) (Olivas Martínez \& Barraza Macías, 2016). De la misma manera la percepción de autoeficacia no refleja necesariamente el rendimiento, es considerado como un factor del éxito académico, la persistencia en diferentes actividades o la organización del tiempo empleado en ellas. Pajares \& Schunk (2001) Al igual que varios estudios han establecido vínculos entre la autoeficacia y la autorregulación con el rendimiento y desempeño, demostrando de esta manera que los alumnos con niveles altos de autoeficacia alcanzaron mejores calificaciones y mayor persistencia en cursos de ciencias e ingeniería en relación con sus compañeros con desconfianza. Por lo tanto, al contar el alumno con habilidades optimas siendo incentivado adecuadamente, las expectativas acerca de la propia eficacia serán un determinante esencial del tipo 
de actividad que escogerá, la cantidad de esfuerza que invierta y el tiempo que dedicará a gestionar las conductas detonantes de estrés (Alegre, 2014).

Según el autor Smith (1989)las dimensiones fundamentales de la autoeficacia son tres: la magnitud, fuerza y generalización. Magnitud de la autoeficacia, va relacionado a todas las pruebas que una persona se siente capaz de superar de manera progresiva en búsqueda de llegar a la meta final. La dimensión, fuerza de la autoeficacia, hace referencia a la confianza con la que una persona enfrenta una situación en particular, influida por las capacidades que sabe posee él como individuo. La tercera dimensión, generalización de la autoeficacia, va relacionada a la capacidad de un individuo para hacer frente a las situaciones de éxito o fracaso de experiencias previas.

\section{Aprendizaje autorregulado}

La propuesta del aprendizaje autorregulado para Rodriguez L, (2008) tiene su origen en norte américa debido a las constantes investigaciones que para algunos destacados autores como Pintrich en 2000, Winne y Hadwin en 1998 y Zimmerman en 1998 y 2000 dicho término se origina por la intensión de querer describir, explicar, controlar y hasta predecir los procesos cognitivos y motivacionales involucrados en los modelos de procesamiento informativo. Para éste fin tuvieron gran relevancia los estudios realizados por Bandura sobre autorregulación relacionándola con la meta cognición. Para Zimmerman \& Pons (1986) y Zimmerman B. (1989) son reconocidos como autorreguladores aquellos estudiantes que participan de forma activa en la obtención de los nuevos conocimientos, y con la visión general del estudiante no podemos vincularlo únicamente con los elementos intelectuales que lo rodean, sino también factores afectivos, emocionales y el equilibrio personal.

En el estudiante autorregulado el conocimiento debe ser adquirido siguiendo estrategias específicas dirigidas a objetivos académicos también específicos; y para tal fin tenemos tres elementos básicos que debemos conocer: (a) las estrategias de aprendizaje dirigidas por los estudiantes, que para Zimmerman \& Pons (1986) son las acciones y aquellos procedimientos que de manera directa nos permitan obtener la información orientada hacia una meta definida, (b) percepciones de autoeficacia del desempeño de las habilidades, que hace referencia a aquellas capacidades que una persona desarrolla para poder organizar de manera correcta y llevar a la práctica mediante la implementación de acciones necesarias para poder llegar a cumplir las tareas propuestas (Bandura, 1986) y (c) las metas académicas, nos indica la manera en que los estudiantes utilizan de forma adecuada las estrategias para lograr alcanzar aquellos objetivos establecidos. Es verdad que el éxito en la realización de las tareas académicas por parte de los estudiantes no es dependiente exclusivamente de ellos; sino, de todo aquello que se encuentra involucrado en éste proceso, y en especial la motivación y la retroalimentación constante mediante procesos de evaluación de las actividades realizadas con anterioridad, es imprescindible tener en cuenta las personas que lo rodean, la motivación que recibe y la evaluación acertada y constante que le permitirá tener una visión real de las capacidades que tiene para poder desempeñar dichas tareas.

\section{Dimensiones de las estrategias de aprendizaje autorregulado}

a) Autorregulación de la Cognición. Bartlett y Piaget identificaron el papel de los esquemas cognitivos en el aprendizaje y la memoria, así como la importancia de la lógica y la coherencia conceptual en la formación de esquemas. De esta manera el proceso cognitivo se encuentra de manera activa en el aprendizaje y el recuerdo, a través de una motivación intrínseca. (Duran Falcón, 2017) 
b) Autorregulación de la Motivación y del Afecto. En el modelo de cognición-motivación de Pitrich y Schrauben, se considera que la relación entre los factores cognitivos y motivacionales influye de manera directa en el compromiso del estudiante con el rendimiento académico y su aprendizaje. (Cardozo, 2008). La motivación es una condición necesaria antes de empezar el proceso de aprendizaje, el querer hacerlo, tener disposición, intención y motivación suficiente, direccionarán los mecanismos cognitivos hacia los objetivos o metas planteadas. (Norabuena, 2011)

c) Autorregulación de Control del ambiente o del Contexto. Pintrich (2000) Dentro de la regulación del contexto considera el conocimiento de los estudiantes acerca de las normas generales y como la forma en la que perciban algunas de ellas puede influir en su enfoque al aula y su aprendizaje en general; así como también el clima de la clase, haciendo referencia a la calidez y el entusiasmo de los maestros, la equidad e imparcialidad para con todos los estudiantes; además de la percepción acerca de la tarea la cual abarca el análisis y percepción, control y evaluación de las mismas. (Lanz, 2006). Para Norabuena (2011) “La gestión del tiempo, el entorno de estudio o la ayuda de profesores y compañeros permitirán al estudiante una mejor adaptación al contexto y posibilita su modificación para adecuarlos a sus objetivos y necesidades propias." (p57)

d) La autorregulación y la estrategia Ejecutiva. Las funciones ejecutivas son las capacidades mentales indispensables para la formulación de metas, planificar formas de cómo lograrlas, llevarlas a cabo y cumplir las metas formuladas en un comienzo. (Lezak, The problem of assessing Executive Functions. , 1982).Al final verifica su actividad comparando los efectos de sus acciones con las intenciones formuladas en un comienzo de tal manera que podrá corregir cualquier error cometido (Norabuena, 2011, pág. 63)

\section{METODOLOGÍA}

Las variables estudiadas fueron la Autoeficacia académica (Output, Input Retroalimentación) y la Autorregulación del aprendizaje (La autorregulación y la estrategia ejecutiva, Autorregulación de la cognición, Autorregulación de la motivación y del afecto y la Autorregulación de control del ambiente o del contexto)

El presente estudio corresponde a una investigación de tipo cuantitativa (Hernandes R., 2014) y con un diseño descriptivo, prospectivo de corte transversal correlacional Ámbito y tiempo Social de la Investigación (Hernandes R., 2014). El estudio se realizó en la Universidad Privada de Tacna en la Facultad de Ciencias de la Salud, en la Escuela de Tecnología Médica en el ciclo 2018-II. Alumno matriculado en el ciclo académico 2018-II de la carrera de Tecnología Médica. La población en el semestre 2018-II equivale a 120 alumnos; de los cuales se consideró solo a los alumnos que aceptaron participar del estudio y consintieron ser parte del estudio, conformada por una muestra de 92 alumnos.

Según la clasificación de Tamayo C. y Silva I. en su trabajo, técnicas e instrumentos de recolección de datos, el instrumento de recolección de datos será la encuesta.

Cuestionario de Expectativas de Autoeficacia Académica Elaborado por Barraza (2010) a continuación se detallará su ficha técnica: el autor es Arturo Barraza Macías Cuenta con 20 ítems, bajo una escala de Likert de 4 puntos, donde 1= nada seguro, 2=poco seguro, 3=seguro y 4= muy seguro. El tiempo de aplicación sería entre 10 - 15 min, su objetivo es medir el nivel de expectativas de autoeficacia académica. Se aplica de forma auto administrada, ya sea de manera individual como colectiva a jóvenes y adultos que se encuentran cursando estudios universitarios. confiabilidad para su aplicación en CPEL -USIL Dado que el instrumento es de origen mexicano, algunos ítems fueron reajustados 
lingüísticamente para la comprensión de los estudiantes de la Universidad donde se hizo el estudio, pasando para su aprobación, por validez de contenido a cargo de 3 especialistas en la temática de la Universidad Nacional Mayor de San Marcos, quienes verificaron la pertinencia, concordancia y coherencia de las afirmaciones propuestas en el cuestionario. Luego de ello, se aplicó un piloto a 30 estudiantes de características similares a la población de estudio, para ver el comportamiento de los ítems y la confiabilidad de los mismos, resultando un valor de $\alpha$ de Cronbach de 0.91 (muy elevado).

Inventario del Aprendizaje autorregulado, el autor Reinhart Lindner, Harris \& Gordon V. 4.01 (1992) Cuenta con 80 ítems, bajo una escala de Likert de 5 puntos, donde 1=Casi siempre típico en mí, 2=Frecuentemente típico en mí, 3=Algunas veces típico en mí, 4= No es muy típico en mí y 5= Es típico en mí en lo absoluto. El tiempo de aplicación es de 20 - 30 min, su objetivo es Medir el nivel de autorregulación para el aprendizaje, se aplica de forma auto administrada, ya sea de manera individual como colectiva a jóvenes y adultos que se encuentran cursando estudios universitarios (de 16 a más años de edad). En el estudio de Durán 2017, se ha realizado el proceso de validación de contenido, en donde se han tenido en cuenta tres aspectos: relevancia, pertinencia y claridad de cada uno de los ítems de los instrumentos, en la que los expertos la consideraron aplicable. Además, se aplicó la prueba estadística de confiabilidad Alfa de Cronbach a una muestra de 20 estudiantes, los datos fueron tabulados en el software estadístico SPSS 21.0 para su control de calidad y procesamiento; en la que salió como resultado igual a 0,823 por lo tanto podemos afirmar que el instrumento que mide dicha variable es confiable.

\section{RESULTADOS}

En la tabla 1 se muestra el nivel de aprendizaje autoregulado alcanzado por el grupo de estudio y los resultados se concentraron en los niveles bajo, medio y muy bajo; donde los porcentajes fueron $6.5 \%$, $50 \%$ y $43.5 \%$ respectivamente

Tabla 1

Nivel de aprendizaje autorregulado

\begin{tabular}{lll}
\hline & Frecuencia & Porcentaje \\
\hline Muy bajo & 0 & $0 \%$ \\
Bajo & 6 & $6.5 \%$ \\
Medio & 46 & $50.0 \%$ \\
Alto & 40 & $43.5 \%$ \\
Muy alto & 0 & $0 \%$ \\
Total & 92 & 100.0 \\
\hline
\end{tabular}

Fuente: elaboración propia

En la tabla 2 se aprecia que la frecuencia de las alternativas marcadas por los alumnos en la dimensión de la estrategia ejecutiva donde No es típico en mí en lo absoluto corresponde al 7\%, No es muy típico en mí 14\%, Algunas veces típico en mí. 36\%, Frecuentemente típico en mí 30\%, Casi siempre típico en mí $13 \%$

La frecuencia de las alternativas marcadas por los alumnos en la dimensión de Autorregulación de la cognición en la cual No es típico en mí en lo absoluto corresponde al 8\%, No es muy típico en mí 17\%, Algunas veces típico en mí. 32\%, Frecuentemente típico en mí 27\%, Casi siempre típico en mí 15\% 
La frecuencia de las alternativas marcadas por los alumnos en la dimensión de autorregulación de la motivación y afecto en la cual No es típico en mí en lo absoluto corresponde al 7\%, No es muy típico en mí 15\%, Algunas veces típico en mí. 35\%, Frecuentemente típico en mí 26\%, Casi siempre típico en mí $16 \%$

La frecuencia de las alternativas marcadas por los alumnos en la autorregulación del ambiente y el contexto en la cual No es típico en mí en lo absoluto corresponde al 6\%, No es muy típico en mí 13\%, Algunas veces típico en mí. 32\%, Frecuentemente típico en mí 32\%, Casi siempre típico en mí $17 \%$.

\begin{tabular}{|c|c|c|}
\hline autorregulación de la estrategia ejecutiva & $\mathrm{n}$ & $\%$ \\
\hline No es típico en mí en lo absoluto. & 7 & $7 \%$ \\
\hline No es muy típico en mí & 13 & $14 \%$ \\
\hline Algunas veces típico en mí. & 34 & $36 \%$ \\
\hline Frecuentemente típico en mí. & 28 & $30 \%$ \\
\hline Casi siempre típico en mí. & 12 & $13 \%$ \\
\hline Total & 92 & $100 \%$ \\
\hline \multicolumn{3}{|l|}{ Autorregulación de la cognición } \\
\hline No es típico en mí en lo absoluto. & 8 & $8 \%$ \\
\hline No es muy típico en mí & 16 & $17 \%$ \\
\hline Algunas veces típico en mí. & 30 & $32 \%$ \\
\hline Frecuentemente típico en mí. & 25 & $27 \%$ \\
\hline Casi siempre típico en mí. & 14 & $15 \%$ \\
\hline Total & 92 & $100 \%$ \\
\hline \multicolumn{3}{|l|}{ Autorregulación de la motivación y afecto } \\
\hline No es típico en mí en lo absoluto. & 7 & $7 \%$ \\
\hline No es muy típico en mí & 14 & $15 \%$ \\
\hline Algunas veces típico en mí. & 33 & $35 \%$ \\
\hline Frecuentemente típico en mí. & 24 & $26 \%$ \\
\hline Casi siempre típico en mí. & 14 & $16 \%$ \\
\hline Total & 92 & $100 \%$ \\
\hline \multicolumn{3}{|l|}{ Autorregulación del ambiente y el contexto } \\
\hline No es típico en mí en lo absoluto. & 6 & $6 \%$ \\
\hline No es muy típico en mí & 12 & $13 \%$ \\
\hline Algunas veces típico en mí. & 29 & $32 \%$ \\
\hline Frecuentemente típico en mí. & 30 & $32 \%$ \\
\hline Casi siempre típico en mí. & 15 & $17 \%$ \\
\hline Total & 92 & $100 \%$ \\
\hline
\end{tabular}

Fuente: elaboración propia

En la tabla 3 se observa los resultados globales de las dimensiones en donde la mínima de la dimensión de estrategia ejecutiva es 20 la máxima 63, la mediana 49.1 y presenta una desviación de 7.7; en la dimensión de cognición la mínima es de 30, la máxima de 63, la media de 48.5, con una desviación de 6.3; para la dimensión de la motivación y el afecto la mínima es de 31, el máximo de 63, la media de 49.2, con una desviación de 7.4; y por último en la dimensión de ambiente y contexto observamos una mínima de 25, máxima de 69, media de 51.1 y una desviación de 8.4 . 
Comparándola con la primera tabla que mostro que el nivel de autorregulación del aprendizaje presente en los estudiantes se mantenía en los niveles alto, medio y bajo; es decir que se guardan similitudes en entre el resultado global de la variable y los resultados por cada dimensión.

Tabla 3

Estadísticos Descriptivos de las dimensiones de autoregulación del aprendizaje

\begin{tabular}{lccccc}
\hline & N & Mínimo & Máximo & Media & Desv. Desviación \\
\hline Autorregulación de la Estrategia ejecutiva & 92 & 20.00 & 63.00 & 49.1087 & 7.76928 \\
Autorregulación de la cognición & 92 & 30.00 & 63.00 & 48.5326 & 6.38069 \\
Autorregulación de la motivación y afecto & 92 & 31.00 & 63.00 & 49.2500 & 7.43266 \\
Autorregulación del ambiente y el contexto & 92 & 25.00 & 69.00 & 51.1848 & 8.43714 \\
N válido (por lista) & 92 & & & & \\
\hline Fuente: elaboración propia & & & & &
\end{tabular}

Esta tabla 4 permite observar el nivel de autoeficacia académica presente en los estudiantes de tecnología médica, en donde el $4 \%$ se considera nada seguro, $22 \%$ poco seguro, $57 \%$ seguro y $17 \%$ muy seguro.

Tabla 4

Nivel de autoeficacia académica

\begin{tabular}{lcc}
\hline & Número & Porcentaje \\
\hline Nada seguro & 4 & $4 \%$ \\
Poco seguro & 21 & $22 \%$ \\
Seguro & 52 & $57 \%$ \\
Muy seguro & 16 & $17 \%$ \\
Total & 92 & $100 \%$ \\
\hline
\end{tabular}

Fuente: elaboración propia

Existe una correlación directa entre autoeficacia académica y el aprendizaje autorregulado en estudiantes (tabla 5).

Tabla 5

Relación entre autoeficacia académica y autorregulación del aprendizaje

\begin{tabular}{llrrr}
\hline & & & $\begin{array}{c}\text { Autorregulación } \\
\text { Académica }\end{array}$ & $\begin{array}{c}\text { Autoeficacia } \\
\text { académica }\end{array}$ \\
\hline Rho de & Autorregulación & Coeficiente de correlación & 1,000 &, $479^{* *}$ \\
Spearman & del aprendizaje & Sig. (bilateral) &. &, 000 \\
& & $N$ & 92 & 92 \\
\cline { 2 - 5 } & Autoeficacia & Coeficiente de correlación &, $479^{* *}$ & 1,000 \\
& académica & Sig. (bilateral) &, 000 &. \\
& $N$ & 92 & 92 \\
\hline
\end{tabular}

**. La correlación es significativa en el nivel 0,01 (bilateral).

Fuente: elaboración propia 


\section{DISCUSIÓN}

La investigación tuvo como objetivo establecer si existe una relación entre autoeficacia académica y la autorregulación del aprendizaje en estudiantes de la carrera de tecnología médica de la universidad Privada de Tacna en el 2018; de mismo modo establecer la relación entre autoeficacia académica y las dimensiones de la autorregulación del aprendizaje (autorregulación de la estrategia ejecutiva, autorregulación de la cognición, autorregulación motivación y afecto, y autorregulación del ambiente y contexto). La mayor limitante que presentó esta investigación fue la participación voluntaria de los estudiantes ya que estos por diversos motivos decidieron prescindir de su participación. En relación al nivel de la autoeficacia académica se encontró que la población se ubicaba en los niveles bajo, medio y alto, con un $6.5 \%, 50 \%, 43.5 \%$ respectivamente sin irse a extremos de la valoración. Con respecto a las dimensiones de la autorregulación del aprendizaje, autorregulación de la estrategia ejecutiva, autorregulación de la cognición, autorregulación de la motivación y afecto, autorregulación del ambiente y el contexto, presentaron una media de 49.1, 48.5, 49.2 y 51.1 respectivamente lo cual nos indica que no hubo extremos y que la población se ubica en el nivel medio de aprendizaje autorregulado

Mientras que el nivel de autoeficacia académica si bien la población se encuentra en todos los niveles, No es típico en mí en lo absoluto corresponde al 7\%, No es muy típico en mí 14\%, Algunas veces típico en mí. 36\%, Frecuentemente típico en mí 30\%, Casi siempre típico en mí 13\%, muestra una distribución parecida a la autorregulación del aprendizaje ya que los porcentajes más altos se encuentran en los niveles intermedios y no en los extremos. Al realizar la prueba de Rho de Spearman mostro una significancia bilateral menor a 0.05, además de un coeficiente de correlación de 0,479 por lo que nos demuestra que existe una relación positiva y moderada entre la autoeficacia académica y la autorregulación en los estudiantes de tecnología médica de la Universidad Privada de Tacna. Al relacionar las dimensiones de autorregulación del aprendizaje con autoeficacia académica se encontraron los siguientes resultados: autoeficacia académica con autorregulación de la estrategia ejecutiva mostro una correlación positiva y moderada (sig. Bilateral 0.01 y coeficiente de correlación 0,449), al relacionarlo con autorregulación de la cognición mostro una correlación positiva y baja (sig. Bilateral 0.01 y coeficiente de correlación 0,325$)$, al relacionarlo con autorregulación de la motivación y afecto mostro una correlación positiva y baja (sig. Bilateral 0.01 y coeficiente de correlación 0,398 ) y al relacionarlo con autorregulación del control del ambiente mostro una correlación positiva y baja (sig. Bilateral 0,01 y coeficiente de correlación 0,385).

Estos resultados coinciden con los resultados presentes en la investigación de Alegre (2014) que encontró una relación positiva y significativa entre la autoeficacia académica y la autorregulación del aprendizaje en los universitarios iniciales de Lima Metropolitana; así mismo Barahona (2014) encontró que la relación entre autoeficacia y autorregulación es altamente significativa y a la vez el nivel de autorregulación es mayor al nivel autoeficacia percibida por los estudiantes y son de suma importancia para un adecuado trabajo académico; y Hendriati (2015) mostro que la autoeficacia, la autorregulación del aprendizaje y los logros académicos están correlacionados positivamente en los estudiantes de la facultad de psicología de Universitas Padjadjaran con lo cual un cambio positivo o negativo influirá de la misma manera en la otra.

Por otro lado Aquino (2015) que también encontró una relación estadísticamente significativa sus resultados difieren ya que encontró que el nivel de autoeficacia era mayor al nivel de autorregulación de los estudiantes de primer ingreso 2015 de la licenciatura de Nutrición del Campus Central, en el 
caso de Hernández (2015) este también encontró una relación positiva y moderada entre ambas variables pero los resultados mostraron que el nivel de autoeficacia académica era menor al nivel de autorregulación del aprendizaje

Considerando los resultados obtenidos podríamos afirmar que cualquier factor positivo o negativo que afecte una de las variables va a repercutir directamente proporcional en la otra, por lo que es necesario que los docentes de todos los cursos promuevan la autoeficacia en los estudiantes y realicen actividades que permitan a los alumnos autorregularse, ya que según Paolini y Bonetto (2013) citado por Aquino (2015), es posible que personas con la misma capacidad y conocimientos obtengan resultados diferentes debido a su nivel de autoeficacia y autorregulación.

Así este estudio concluye en que existe una relación directa entre autoeficacia académica y el aprendizaje autorregulado en los estudiantes de la carrera de Tecnología Médica de la Universidad Privada de Tacna en el año 2018 ya que la prueba Rho de Spearman evidenció una correlación positiva moderada de 0,449 ( $p$ valor 0,01).

Considerando los resultados respecto al nivel de autorregulación del aprendizaje y autoeficacia académica es de considerarse positivo y destacable, pero se debe tener en cuenta también se presentan estudiantes en un nivel bajo, por lo que se propone a la dirección de escuela la implementación de un monitoreo periódico de estas dos variables y de igual manera capacitar a los docentes para que logren identificar los factores académicos y socioemocionales, ya que si bien se han obtenido buenos resultados se debe hacer todo lo necesario para mantenerlo y /o mejorarlo. Se sugiere a la escuela de tecnología médica una revisión acerca de cómo se está llevando el curso y sobre si los alumnos están tomando la importancia debida al curso en cuestión. Se sugiere a los docentes incentivar en clase estrategias de repaso, elaboración de nueva información y su organización. Se recomienda a los docenes instruir a los alumnos sobre la utilización de estrategias como el lenguaje auto dirigido, auto recompensas al lograr ciertos avances académicos; y resaltar la importancia de la tarea con su importancia en el futuro dentro del aula. Se propone a los docentes implementar dentro del salón estrategias dirigidas a mejorar la gestión del tiempo, así como también el trabajo colaborativo e individual, de igual manera consultar a los alumnos acerca de los ambientes proporcionados para el desarrollo de sus actividades academias fuera de las aulas y coordinar con la comunidad educativa la posibilidad de modificar y/o incrementar los ambientes de manera adecuada.

\section{REFERENCIAS BIBLIOGRÁFICAS}

Alegre, A. (2014). Autoeficacia académica, autorregulación del aprendizaje y rendimiento académico en estudiantes universitarios iniciales. Propósitos y Representaciones, 79-120.

Bandura, A. (1986). Fundamentos sociales del pensamiento y la acción: Una teoría social cognitiva. NJ: PrenticeHall.

Bandura, A. (1987). Pensamiento y acción. Fundamentos sociales. Barcelona.

Bandura, A. (1997). "Guía para la Construcción de Escalas de Autoeficacia".

Barahona Arévalo, M. (2014). "Correlaión entre autoeficacia, autorregulación del aprendizaje y rendimiento academico en los estudiantes de 4to y 5to bachillerato del colegio San Francisco Javier de la Verapaz (Tesis de Maestría). Universidad Rafael Landívar, Guatemala de la Asunción.

Barraza, M. (2010). Validación del inventario de expectativas de autoeficacia académica en tres muestras secuenciales e independientes. . Revista de Investigación Educativa, 1-30.

Cardozo, A. (2008). Motivación, aprendizaje y rendimiento académico en estudiantes del primer año universitario. Laurus, 209-237. 
Duran Falcón, J. (2017). Aprendizaje autorregulado e inteligencia emocional de las estudiantes de educación inicial 2016 de la IESPP "Emilia Barcia Boniffatti" (Tesis de Maestría). Universidad César Vallejo.

González-Torres, M., \& Tourón, J. (1992). Autoconcepto y rendimiento escolar: sus implicaciones en la motivación y en la autorregulación del aprendizaje. Pamplona: EUNSA.

Lanz, M. (2006). El aprendizaje autorregulado. Enseñar a aprender en diferentes entornos educativos.

Lezak, M. (1982). The problem of assessing Executive Functions. . International Journal of Psychology, 281 - 297.

Norabuena, R. (2011). Relación entre el aprendizaje autorregulado y rendimiento académico en estudiantes de enfermería y obstetricia de la Universidad Nacional "Santiago Antúnez de Mayolo" - Huaraz (Tesis de pre grado). Universidad Nacional Mayor de San Marcos, Lima.

Olivas Martínez, A., \& Barraza Macías, A. (2016). Expectativas de autoeficacia académica en alumnos de un bachillerato técnico de la ciudad de Durango. (U. P. Durango, Ed.) Mexico.

Pajares , F., \& Schunk, D. (2001). Self-Beliefs and School Success: Self-Efficacy, Self-Concept, and School Achievement. International Perspectives on Individual Differences: Self-Perception, 239-265.

Pintrich, P. (1986). Comprender el aprendizaje autorregulado. San Francisco: Jossey-Base. : P. R. Pintrich (ED).

Pintrich, P. (2000). The role of goal orientation in self-regulated learning. San Diego: Academic Press.

Pintrich, P. (2002). The Role of Metacognitive Knowledge in Learning, Teaching, and Assessing. Theory Into Practice - THEORY PRACT, 219-225.

Pintrich, P., Mckeachie, W., Lin, G., \& Smith, D. (1986). Motivated strategies for learning questionnaire. Michigan: The University of Michigan.

Pool-Cibrián, W. (2013). Autoefiacia y uso de estrategias para el aprendizaje autorregulado en estudiantes universitarios . Revista Electrónica de Investigación Educativa, 21-37.

Roces, C., \& González-Pineda, J. (1999). Relaciones entre motivación, estrategias de aprendizaje y rendimiento académico en estudiantes universitarios. Revista Electrónica del Departamento de Psicología.

Rodriguez, L. (2008). Variables cognitivo-motivacionales, comportamentales y contextuales y su relación con los procesos de autorregulación del aprendizaje en área de las matemáticas (Tesis). Universidad de Oviedo, Oviedo.

Schunk, H., \& Zimmerman, B. (1994). Autorregulación en la Educación: Retrospectiva y prospectiva. N. J.: Eribaum. .

Smith, A. (1989). A review of the effects of noise on human performance. Scandinavian Journal of Psychology, 185-206.

Valverde Riascos, O. O. (2011). Las creencias de autoeficacia en la práctica pedagógica del docente universitario de humanidades, ciencias sociales, educación y ciencias contables, económicas y administrativas (Tesis de Doctorado). Universidad de Valencia.

Zimmerman, B. (1989). Los modelos de aprendizaje Autorregulado y Los Logros académicos. New York: SpringerVerlang, 1-25.

Zimmerman, B. (2000). Attaining self regulation: a social cognitive perspective. Handbook of Self - Regulation, 13-38.

Zimmerman, B., \& Pons, M. (1986). Development of a structured interview for assessing student use of selfregulated learning strategies. American educational research journal, 614-628. 